\title{
ESTUDIO DE LAS CÓNICAS EN ALGUNAS MÉTRICAS: PROPUESTA PARA EL DESARROLLO DEL PENSAMIENTO ESPACIAL
}

\section{STUDY OF CONICS IN SOME METRICS: PROPOSAL FOR THE DEVELOPMENT OF SPATIAL THINKING}

\author{
Jesús Adrián Antonio Peña ${ }^{1}$ \\ jesus.antonioluuptc.edu.co \\ Cristian Julián Garzón Zipa ${ }^{2}$ \\ cristian.garzonduptc.edu.co \\ Omaida Sepulveda Delgado ${ }^{3}$ \\ omaida.sepulvedaAuptc.edu.co
}

Universidad Pedagógica y Tecnológica de

Colombia (Tunja, Colombia)

\begin{abstract}
1 Docente, Licenciado en Matemáticas, estudiante de Maestría en Educación Matemática, Universidad Pedagógica y Tecnológica de Colombia, Tunja, Colombia. Email: jesus.antonio@uptc.edu.co Phone: +573124884925 ORCID ID: https://orcid.org/0000-0002-7685-0023

2 Docente, Licenciado en Matemáticas, estudiante de Maestría en Educación Matemática, Universidad Pedagógica y Tecnológica de Colombia, Tunja, Colombia. Email: cristian.garzon@uptc.edu.co Phone: +573214337938 ORCID ID: https://orcid.org/0000-0001-8171-2621

3 Docente Investigadora, Licenciada en Matemáticas, Ingeniera de Sistemas, Especialista en computación para la docencia, Magister en Ciencias Matemáticas y Doctora en Ciencias de la educación, Universidad Pedagógica y Tecnológica de Colombia, Tunja, Colombia. Email: omaida. sepulveda@uptc.edu.co Phone: +573118556222 ORCID ID: https://orcid.org/0000-0002-2950-8137
\end{abstract}

\section{RESUMEN}

Las cónicas son trabajadas esencialmente desde su representación en la métrica Usual (euclidiana) dejando de lado las diferentes representaciones que se pueden obtener al utilizar otras formas de medida; el objetivo principal de este artículo fue sintetizar los resultados más importantes del trabajo investigativo de Antonio y Garzón (2017) referente al estudio geométrico y analítico de las cónicas cuando se abordan desde otras 
métricas (taxi, máximo y discreta) y reflexionar acerca de la forma en que se está enseñando esta temática, ya que desde el Ministerio de Educación Nacional hay una preocupación por el detrimento del pensamiento espacial y hay una necesidad de rescatar los análisis de tipo intuitivo y crítico en los estudiantes. La investigación se realizó bajo el enfoque teórico-documental donde se tomaron como principales referentes algunas propuestas teóricas y didácticas de las cónicas desde la métrica del taxi; el trabajar las cónicas con distintas métricas permite explorar nuevas formas de medir y promueve el desarrollo del pensamiento geométrico-espacial a través de diferentes situaciones que confrontan el conocimiento del estudiante, además, desde la teoría de los registros de representación resulta ser una estrategia favorable pues "la comprensión no significa dar un salto desde el contenido de una representación hasta el concepto puramente matemático representado, sino en relacionar diversos contenidos de representación del mismo concepto" (Duval, 2006, p. 158). En muchas ocasiones se asocia una cónica con una representación gráfica determinada, en esta investigación se lograron nuevas representaciones tanto algebraicas como geométricas de las cónicas cuando se cambia la forma de medir, con esto entendemos mejor la definición de cada cónica como lugar geométrico, dejando claro que hay varios caminos para abordar las cónicas en un proceso de enseñanza escolar que favorecen el desarrollo del pensamiento espacial.

PALABRAS CLAVE: Cónicas, Métricas, Lugar Geométrico, Registros de Representación, Pensamiento Espacial.

\section{ABSTRACT}

Conics are essentially worked from their representation in the Usual metric (Euclidean) leaving aside the different representations that can be given when using other forms of measurement; the main objective of this article was to synthesize the most important results of the research work of Antonio and Garzón (2017) on the geometric and analytical study of conics when they are approached from other metrics (taxi, maximum and discrete) and to think on the way in which this subject is being taught. The Ministry of National Education of Colombia has a concern for the detriment of spatial thinking and there is a need to rescue the intuitive and crtitical analyses types in students. The research was carried out under the theoreticaldocumentary approach where some theoretical and didactic proposals of the conics from the taxi metrics were taken as the main reference; working with conics with different metrics allows exploring new ways of measuring and promotes the development of geometric-spatial thinking throughout different situations that confrontates the student's knowledge. Furthermore, from the theory of representation registers it turns out to be a favorable strategy because "understanding does not mean making a leap from the content of a representation to the purely mathematical concept represented but in relating diverse contents of representation of the same concept" (Duval, 2006, p. 158). On many occasions, a conic is associated with a specific graphic representation. In this research, new algebraic and geometric representations of conics were achieved when the way of measuring is changed, and with this we better understand the definition of each conic as a geometric place, making it clear that there are several ways to approach conics in a school teaching process that favour the development of spatial thinking.

KEY WORDS: Conics, Metrics, Geometric Place, Representation Records, Spatial Thinking.

\section{INTRODUCCIÓN}

El aprendizaje de las cónicas dentro del marco de la educación media colombiana está enfocado como "Explora y describe las propiedades de los lugares geométricos y de sus transformaciones a partir de diferentes representaciones" 
(Derechos Básicos de Aprendizaje -DBAMinisterio de Educación Nacional -MEN- 2016, p. 78), en los DBA hay un interés por desarrollar la competencia espacial ya que hay un "detrimento de la geometría elemental y el pensamiento espacial [así como] ausencia de actividades y problemas interesantes" (MEN, 1998, p. 5), y esto se puede explicar porque:

Las reformas de las matemáticas escolares [...] eliminaron la geometría como curso paralelo al algebra; relegaron los temas geométricos para el final de los programas [...] y trataron de remplazar las pruebas de tipo sintético por elegantes pruebas de tipo algebraico. (Vasco, 2006, p.27)

A raíz de esto se dio a las matemáticas básicas un exagerado manejo estructural y trajo consigo muchas consecuencias negativas, es por esto que "se empezó a rescatar el valor de lo empírico y de lo intuitivo en los procesos de construcción del conocimiento matemático en la escuela." (MEN, 1998, p. 16). Trasladando esta cuestión a las Cónicas y teniendo en cuenta la riqueza en propiedades geométricas que estas figuras tienen, permitirían ser trabajadas no solo desde el enfoque algebraico y estructural, también se podrían buscar estrategias de trabajo donde se priorice el sentido intuitivo sin dejar de lado las demás propiedades matemáticas de este tipo de figuras, pero ¿Cómo lograr este aprendizaje?

Por ejemplo, una forma de priorizar el concepto de lugar geométrico de las cónicas seria con el tratamiento de otras métricas, la presente investigación está referida especialmente a dicho tratamiento, donde se mostrará un contraste de las diferentes expresiones tanto algebraicas como geométricas de las cónicas cuando su definición como lugar geométrico en no solo se moviliza desde la métrica euclidiana (usual) si no desde otras métricas (taxista, máximo y discretal. La elección de estas métricas se debe a la simplicidad de sus definiciones y a su aplicabilidad en problemas que requieren de procesos de razonamiento, modelación, comunicación y ejercitación de procedimientos.

El artículo se realizó bajo el enfoque teóricodocumental donde se tomó como principal referente el estudio de Antonio y Garzón (2017), al igual que otros estudios como los de Valdivia \& Parraguez (2012), Bonilla (2012), Izquierdo \& Ardila (2013), Bonilla, González \& Chavarro (2014) y Loiola \& Costa (2015) que sustentan propuestas teóricas y didácticas para la comprensión de las cónicas desde otras métricas diferentes a la métrica usual.

El trabajar las cónicas con distintas métricas permite explorar nuevas formas de medir y promueve el desarrollo del pensamiento geométrico-espacial a través de diferentes situaciones que confrontan el conocimiento del estudiante, además de ser una forma de trabajarlas desde su definición como lugar geométrico, para lograr esto "el profesor debe imaginar y proponer a los alumnos situaciones que puedan vivir y en las que los conocimientos van a aparecer como la solución óptima y descubrible en los problemas planteados" (Brousseau citado por MEN, 1998, p. 96).

\section{EL CONCEPTO DE DISTANCIA}

Para poder abordar diferentes formas de medir el concepto clásico de distancia llongitud de segmento de recta que une dos puntos) debe ser ampliado, en Munkres (2002) una distancia en un conjunto no vacío, es una función donde a una pareja de elementos del conjunto les corresponde un valor numérico real, dicho valor es la distancia y cumple las siguientes tres propiedades:

- Positividad. La distancia es un valor mayor o igual a cero, la igualdad se da cuando los dos elementos son iguales. 
- Simetría. La distancia del primer elemento al segundo será igual que del segundo al primero.

- Desigualdad triangular. Dados tres elementos del conjunto, la distancia del primer elemento al tercer elemento debe ser menor o igual a la suma de las distancias del primer elemento al segundo y del segundo elemento al tercero.

A partir de las condiciones que debe cumplir una función para denominarse distancia, hay un gran compendio de ejemplos de métricas, para este caso se trabajaran la métrica usual, del taxista, del máximo y la métrica discreta en el conjunto de $\mathrm{R}^{2}$.

\section{MÉTRICA USUAL}

Sean: $A=\left(x_{1}, y_{1}\right)$ y $B=\left(x_{2}, y_{2}\right)$ :dos elementos que pertenecen a $\mathrm{R}^{2}$, se denota la función distancia en la métrica usual como $\boldsymbol{d}_{\boldsymbol{u}}$, y se define de la siguiente forma $\quad d_{u}\left(A_{n} B\right)=$ $\sqrt{\left(x_{2}-x_{1}\right)^{2}+\left(y_{2}-y_{1}\right)^{2}}$, : esta es la fórmula más usada para calcular la distancia entre dos elementos (puntos) en $\mathrm{R}^{2}$.

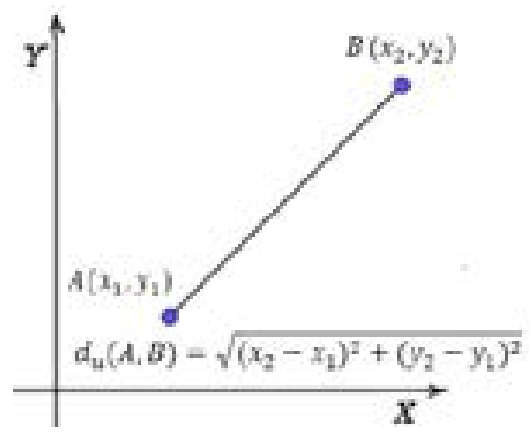

Figura 1. La Distancia entre dos puntos con la métrica usual en $\mathrm{R}^{2}$ es la magnitud del segmento de recta que une a los dos puntos.

Fuente: Los autores.

\section{MÉTRICA DEL TAXISTA}

Sean: $A=\left(x_{1}, y_{1}\right) y \quad B=\left(x_{2}, y_{2}\right)$ dos elementos que pertenecen a $\mathrm{R}^{2}$, se denota la función distancia en la métrica taxista como $d_{t}$, y se define de la siguiente forma

$$
d_{t}(A, B)=\left|x_{2}-x_{1}\right|+\left|y_{2}-y_{1}\right|
$$

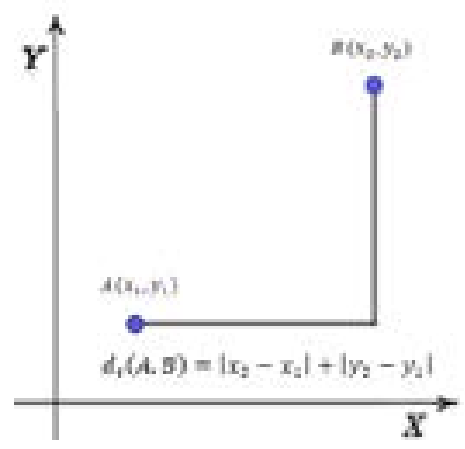

Figura 2. La Distancia entre dos puntos con la métrica del taxista en $\mathrm{R}^{2}$ es la suma de las magnitudes de los dos segmentos de la imagen Fuente: Los autores.

\section{MÉTRICA DEL MÁXIMO}

Sean: $A=\left(x_{1}, y_{1}\right)$ y $B=\left(x_{2}, y_{2}\right)$ dos elementos que pertenecen a $R^{2}$, se denota la función distancia en la métrica del máximo como $d_{M}$, y se define de la siguiente forma $d_{M}(A, B)=$ máx $\left\{\left|x_{2}-x_{1}\right|,\left|y_{2}-y_{1}\right|\right\}$, máx significa mas grande entre los dos posibles.

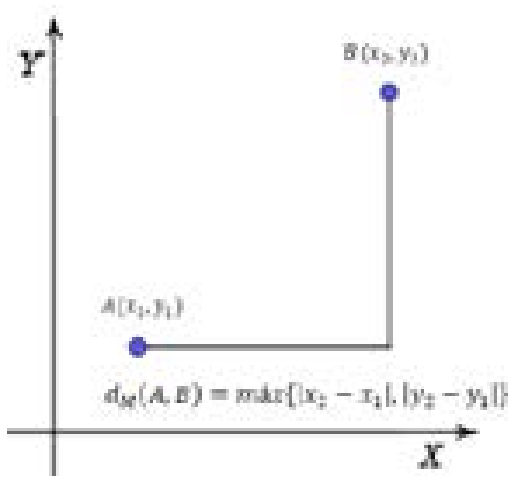

Figura 3. La Distancia entre dos puntos con la métrica del máximo en $\mathrm{R}^{2}$ es la mayor magnitud de los dos segmentos de la imagen. Fuente: Los autores.

\section{MÉTRICA DISCRETA}

Sean: $A=\left(x_{1}, y_{1}\right)$ y $B=\left(x_{2}, y_{2}\right)$ y dos elemen- 
tos que pertenecen a $\mathrm{R}^{2}$, se denota la función distancia en la métrica discreta como $d_{d}$, y se define de la siguienteforma:

$d_{d}(A, B)=\left\{\begin{array}{l}1 \text { si }\left(x_{1}, y_{1}\right) \neq\left(x_{2}, y_{2}\right) \\ 0 \text { si }\left(x_{1}, y_{1}\right)=\left(x_{2}, y_{2}\right)\end{array}\right.$.

de lo anterior la distancia es si son elementos distintos y si son elementos iguales.

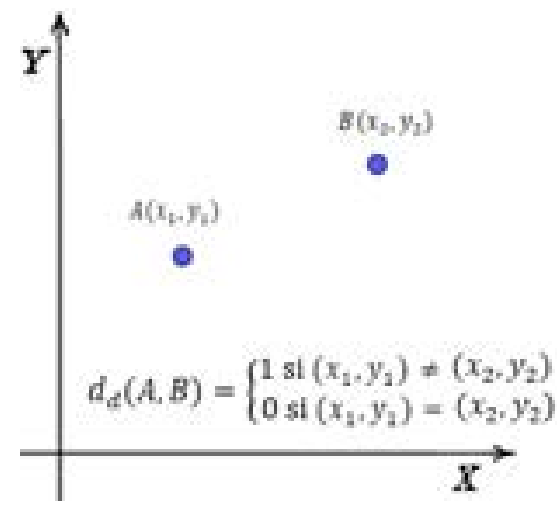

Figura 4. La Distancia entre dos puntos con la métrica discreta en $\mathrm{R}^{2}$ es de magnitud uno si los puntos son diferentes y de magnitud cero si los puntos son iguales.

Fuente: Los autores.

\section{SECCIONES CÓNICAS}

Recurriendo a las definiciones presentadas por Lehmann (1992), las cónicas pueden ser descritas mediante sus lugares de geometría en el plano como:

Definición 1. "La circunferencia es el lugar geométrico de un punto que se mueve en un plano de tal manera que se conserva siempre una distancia constante a un punto fijo de ese plano" (p. 99), dicho punto se denotará como $C$ y será llamado el centro de la circunferencia, $P$ un punto móvil y $r$ el valor constante y positivo al que llamaremos radio de la circunferencia. Por lo anterior se debe cumplir la siguiente expresión:

$d(C, P)=r$

Definición 2. "La parábola es el lugar geométrico de un punto que se mueve en el plano de tal manera que su distancia de una recta fija, situada en el plano, es siempre igual a su distancia de un punto fijo del plano y que no pertenece a la recta." (p. 149). El punto fijo se denotará $F$ y será llamado foco de la parábola, la recta fija se denotará $l$ y será llamada directriz, y el punto móvil del plano que equidista de $F$ y $l$ será llamado $P$. Por lo anterior se puede describir la definición de parábola como:

$d(F, P)=d(P, l)$

Definición 3. "La elipse es el lugar geométrico de un punto que se mueve en un plano de tal manera que la suma de sus distancias a dos puntos fijos es siempre igual a una constante, mayor que la distancia entre los dos puntos." (p. 173). Los dos puntos fijos los denotaremos $F_{1}$ y $F_{2}$ y serán llamados focos de la elipse, $P$ será el punto móvil del plano y $k$ será la constante positiva mayor que la distancia entre los dos focos, según la definición de elipse como lugar geométrico tenemos la siguiente ecuación:

$d\left(F_{1}, P\right)+d\left(F_{2}, P\right)=k ; k>d\left(F_{1}, F_{2}\right)$

Definición 4. "La hipérbola es el lugar geométrico de un punto que se mueve en un plano de tal manera que el valor absoluto de la diferencia de sus distancias a dos puntos fijos del plano, llamados focos, es siempre una cantidad constante, positiva y menor que la distancia entre los focos." (p. 191). El punto que se mueve en el plano será llamado $P$, los dos puntos fijos del plano serán denotados $F_{1}$ y $F_{2}$, y la cantidad constante se denotara $k$, por lo anterior se debe cumplir la siguiente expresión:

$\left|d\left(F_{1}, P\right)-d\left(F_{2}, P\right)\right|=k ; 0<k<d\left(F_{1}, F_{2}\right)$

Definición 5. La distancia de un punto $P$ a una recta $l$ resulta ser la mínima distancia que existe entre dicho punto y un punto de la recta en cuestión. Siguiendo los pasos que exponen Loiola \& Costa (2015) la distancia mínima de un punto a una recta será el radio de la circunferencia tangente a la recta dicha circunferencia está centrada en $P$. (Este método para hallar la 
distancia será muy útil en la definición de la parábola en las métricas del taxista y máximol.

Para la métrica usual la distancia mínima de un punto $P$ de coordenadas $(a, b)$ a una recta $l$ de la forma $A x+B y+C=0$, esta dado por:

$$
d_{u}(P, l)=\frac{|A(a)+B(b)+C|}{\sqrt{A^{2}+B^{2}}}
$$

\section{METODOLOGÍA}

Este trabajo se realizó por medio del enfoque documental donde se sintetizo los principales resultados de la investigación de Antonio y Garzon (2017) y se tomaron como otros referentes los estudios y propuestas didácticas desarrolladas con el uso de métricas en especial con la métrica del taxista [Valdivia \& Parraguez (2012), Bonilla (2012), Izquierdo \& Ardila (2013), Bonilla et al. (2014) y Loiola \& Costa (2015)] así como textos conocidos en el estudio de la geometría analítica y las métricas (Lehmann (1992) y Munkres (2002)); con estos antecedentes y referentes teóricos, se prosiguió con una inspección de la información para así poder generar los resultados que expone este artículo.

En el desarrollo del trabajo se introdujo el concepto de lugar geométrico de cada una de las cónicas en diferentes métricas y se muestran los resultados obtenidos al realizar el estudio analítico y gráfico en la métrica usual, taxista, máximo y discreta, para finalmente contrastar los resultados desde la teoría de los registros de representación, resaltando las bondades que puede generar esta propuesta en el aprendizaje de las cónicas en la matemática escolar pues la actividad matemática desarrollada en la presente investigación es un ejemplo de cómo abordar de una manera distinta la temática de las cónicas priorizando los criterios geométricos y el pensamiento intuitivo.

\section{RESULTADOS: CÓNICAS EN LAS DIFERENTES MÉTRICAS}

\section{Cónicas en la métrica usual.}

Circunferencia. De acuerdo a la Definición 1, los puntos $P=(x, y)$ y $C=(h, k)$ deben satisfacer la expresión (1), por lo que se tiene en la métrica usual:

$$
d_{u}(C, P)=\sqrt{(x-h)^{2}+(y-k)^{2}}
$$

Ahora la anterior distancia debe ser igual a un valor positivo constante por lo tanto, se tiene:

$$
\sqrt{(x-h)^{2}+(y-k)^{2}}=r
$$

La anterior expresión corresponde al lenguaje algebraico de la circunferencia en la métrica usual, su representación gráfica seria:

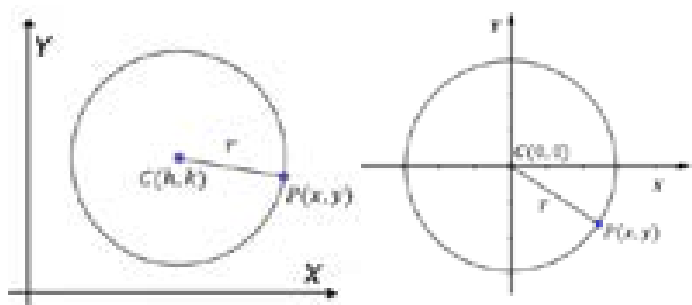

Figura 5. Representación de circunferencias en la métrica usual Fuente: Los autores.

Parábola. Por la Definición 2, asumiendo que la directriz $l$ es de la forma $A x+B y+C=0$ y el foco $F$ es de coordenadas $(a, b)$, por la métrica usual, la distancia entre los puntos $F=(a, b)$ y $P=$ $(x, y)$ es:

$$
d_{u}(F, P)=\sqrt{(x-a)^{2}+(y-b)^{2}}
$$

mientras que la distancia de $P$ a la directriz $l$, usando la ecuación de distancia de un punto a una recta para la métrica usual expresada en la Definición 2 queda:

$$
d u(P, l)=\frac{|A x+B y+C|}{\sqrt{A^{2}+B^{2}}}
$$

por lo anterior, si se iguala $d_{u}(F, P)$ y $d_{u}(P, l)$ se obtiene la expresión algebraica general de la parábola para le métrica usual:

$$
\sqrt{(x-a)^{2}+(y-b)^{2}}=\frac{|A x+B y+C|}{\sqrt{A^{2}+B^{2}}}
$$


En el lenguaje grafico la parábola para la métrica usual es la siguiente:

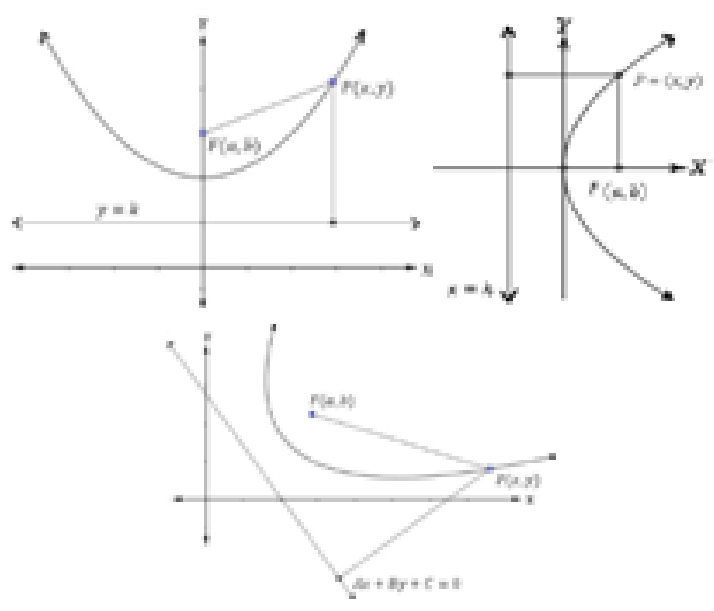

Figura 6 Parábola en la métrica usual.

Fuente: Los autores.

Elipse. Por la definición 3 , Sean $F 1=(a, b)$ y $F 2=$ $(c, d)$ los focos de la elipse, y $P=(x, y)$ cualquier punto que pertenece a la elipse, se tiene lo siguiente:

Distancia de cualquier punto de la elipse al primer foco $F_{1}$ :

$$
d_{u}\left(F_{1}, P\right)=\sqrt{(x-a)^{2}+(y-b)^{2}}
$$

Distancia de cualquier punto de la elipse al segundo foco $F_{2}$ :

$$
d_{u}\left(F_{2}, P\right)=\sqrt{(x-c)^{2}+(y-d)^{2}}
$$

aplicando la expresión algebraica (3) se tiene:

$$
\begin{gathered}
d\left(F_{1}, P\right)+d\left(F_{2}, P\right)=k ; k>d\left(F_{1}, F_{2}\right) \\
\sqrt{(x-a)^{2}+(y-b)^{2}}+\sqrt{(x-c)^{2}+(y-d)^{2}}=k
\end{gathered}
$$

La siguiente figura representa la ecuación de la elipse

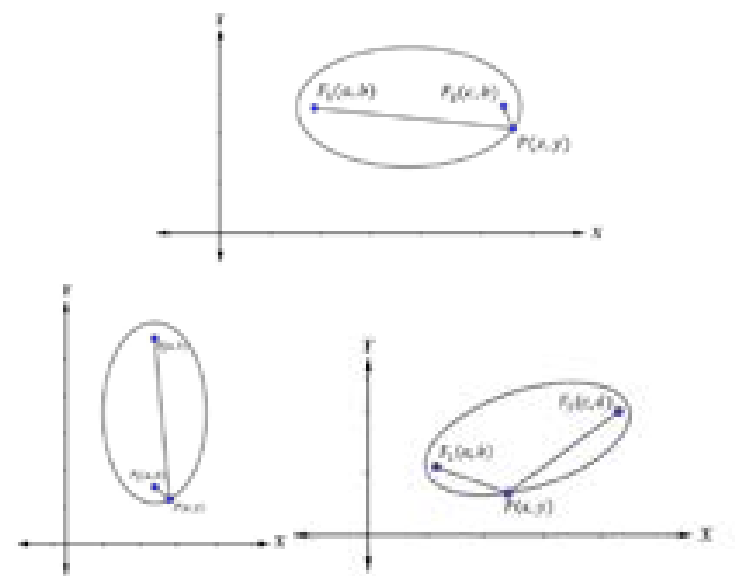

Figura 7. Elipse en la métrica usual.

Fuente: Los autores.

Hipérbola. Por la definición 4 se asume lo siguiente, sean $F 1(a, b)$ y $F 2(c, d)$ focos de la hipérbola, la distancia de un punto $P=(x, y)$ a los dos focos en la métrica usual está dada por:

$$
\begin{aligned}
& d_{u}\left(F_{1}, P\right)=\sqrt{(x-a)^{2}+(y-b)^{2}} \\
& d_{u}\left(F_{2}, P\right)=\sqrt{(x-c)^{2}+(y-d)^{2}}
\end{aligned}
$$

Aplicando la expresión algebraica (4) se obtiene la ecuación general de la hipérbola en la métrica usual:

$$
\begin{gathered}
\left|d\left(F_{1}, P\right)-d\left(F_{2}, P\right)\right|=k ; 0<k<d\left(F_{1}, F_{2}\right) \\
\left|\sqrt{(x-a)^{2}+(y-b)^{2}}-\sqrt{(x-c)^{2}+(y-d)^{2}}\right|=k
\end{gathered}
$$


La figura que representa la ecuación es:
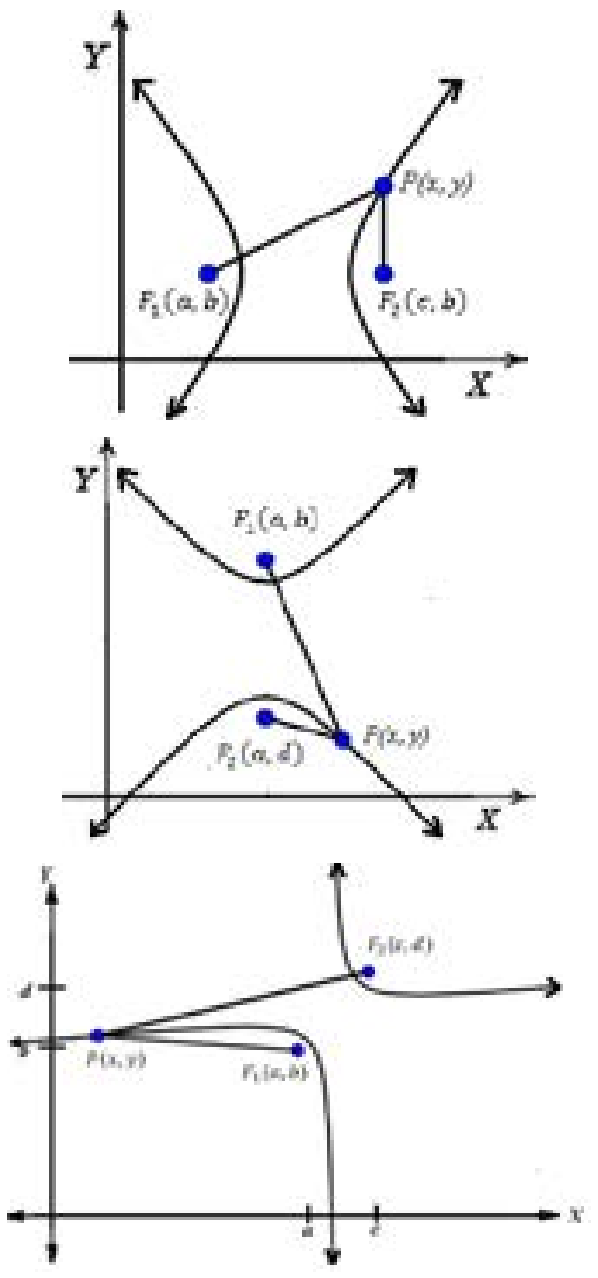

Figura 8. Hipérbola en la métrica usual. Fuente: Los autores.

\section{CÓNICAS EN LA MÉTRICA DEL TAXISTA}

Circunferencia. Para mostrar el comportamiento de la circunferencia en la métrica del taxista, se recurre a la Definición 2, tomamos $C=(h, k)$ el centro de la circunferencia y $P=(x, y)$ un punto en $\mathrm{R}^{2}$ que pertenecerá a la circunferencia. De acuerdo a este tipo de métrica la distancia entre el punto $C$ y el punto $P$, está dado por la siguiente expresión:

$$
d_{t}(C, P)=|x-h|+|y-k|,
$$

igualando la ecuación anterior con la expresión (1) expuesta en la Definición 2, la ecuación de la circunferencia con centro en $C=(h, k)$ y radio en la métrica del taxista está dada por la expresión:

$$
|x-h|+|y-k|=r
$$

la gráfica que representa esta ecuación es:
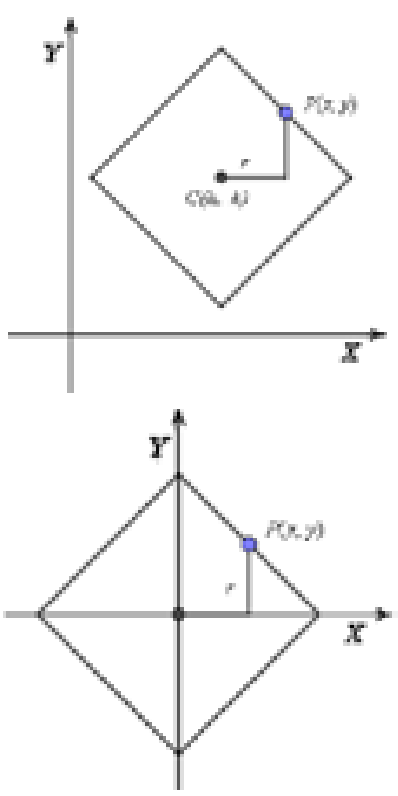

Figura 9. Circunferencias en la métrica del taxista.

Fuente: Los autores.

Parábola. A partir de la Definición 2, se debe establecer inicialmente la distancia de un punto a una recta en la métrica del taxista, para esto se tiene a $l$ una recta de la forma $A x+B y+C=0$ con un punto $M \in l$, y se estudia su distancia a un punto cualquiera $P$ de coordenadas $(x, y)$, para esto se recurre al método de las circunferencias mencionado en la Definición 5 (Se realizara una reconstrucción más detallada basándose en los resultados de Loiola \& Costa (2015)):

Empezamos trazando distintas circunferencias (definidas en la métrica del taxista) concéntricas en $P$ que irán aumentando progresivamente el radio, hasta encontrar la primera circunferencia que se intercepte con la recta $l$.

El radio de dicha circunferencia resulta ser la mínima distancia del punto $P$ a la recta $l$ con la métrica del taxista; puede haber solo un punto de intersección (caso I), o varios puntos de intersección (caso II). 


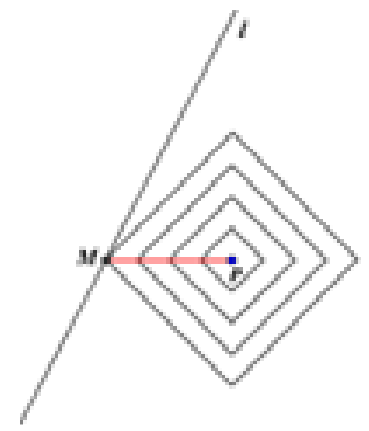

Figura 10. Caso I. Distancia mínima del punto $P$ a la recta $l$ en la métrica del taxista.

Fuente: Los autores.

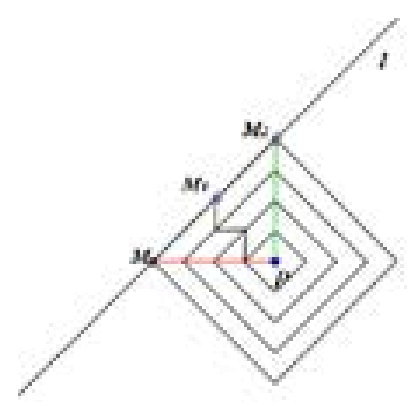

Figura 11. Caso II. Intercepción de una circunferencia con una recta en más de un punto. Fuente: Los autores.

Como se puede observar, para el caso I, solo existe un camino para poder medir la distancia mínima que hay entre el punto $P$ y la recta $l$, en cambio para el caso II, existen infinitos caminos para poder determinar la distancia mínima del punto a la recta, para facilitar el método, solo se asumirán dos caminos, aquel que va del punto $P$ y se desplaza de forma vertical hacia la recta, y el otro, que parte del punto y se desplaza de forma horizontal hasta llegar a la recta, por tanto, todo se resumirá a conocer cuál es la mínima longitud de los dos caminos mencionados, sin importar el caso que se trabaje.

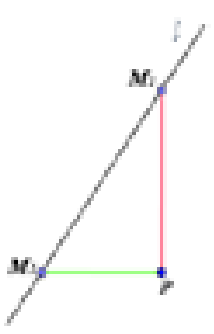

Figura 12. La distancia mínima del punto a la recta, será la menor longitud de los dos segmentos que conectan al punto con la recta.

Fuente: Los autores.

Para obtener una expresión analítica a esta distancia, se toma el punto concéntrico $P$ y se trazan las rectas $X=x$ y $Y=y$, nombrándose $X_{I}$ y $X_{2}$, los puntos de intersección entre la recta $l y$ las rectas $X=x$ y $Y=y$ respectivamente.

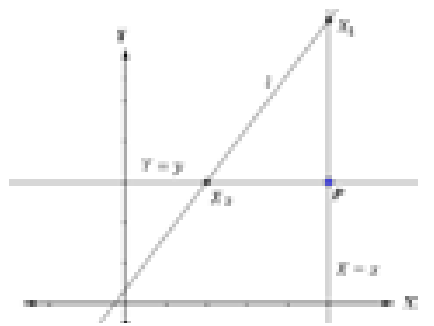

Figura 13. Rectas $X=x \& Y=y$ que pasan por e interceptan a la recta $l$

Fuente: Los autores.

Se definen las parejas ordenadas de $X_{1}$ y $X_{2}$ de la siguiente forma:

- Para $X_{1}$, se sabe que su abscisa es el valor $x$, el valor de su ordenada se obtendría remplazando el valor de su abscisa en la ecuación de la recta $l$ de la siguiente forma:

$$
\begin{gathered}
A x+B y+C=0, \\
y=-\frac{A x}{B}-\frac{C}{B},
\end{gathered}
$$

Por tal razón:

$$
X_{1}=\left(x,-\frac{A x}{B}-\frac{C}{B}\right)
$$

- De forma análoga:

$$
X_{2}=\left(-\frac{B y}{A}-\frac{C}{A}, y\right)
$$


Luego, la distancia de $d_{t}\left(P, X_{1}\right)$ es:

$$
d_{t}\left(P, X_{1}\right)=|x-x|+\left|y-\left(-\frac{A x}{B}-\frac{C}{B}\right)\right|=\left|\frac{A x+B y+C}{B}\right|
$$

la distancia de $d_{t}\left(P, X_{2}\right)$ es:

$$
\begin{gathered}
d_{t}\left(P, X_{2}\right)=|x-x|+\left|y-\left(-\frac{A x}{B}-\frac{C}{B}\right)\right|=\left|\frac{A x+B y+C}{B}\right| \\
d_{t}\left(P, X_{2}\right)=\left|x-\left(-\frac{B y}{A}-\frac{C}{A}\right)\right|+|y-y|=\left|\frac{A x+B y+C}{A}\right|
\end{gathered}
$$

Por tanto:

$$
d_{t}(P, l)=\min \left\{\left|\frac{A x+B y+C}{B}\right|,\left|\frac{A x+B y+C}{A}\right|\right\}, A \neq 0, B \neq 0,
$$

Por lo anterior se tiene:

$$
d_{t}(P, l)=\frac{|A x+B y+C|}{\operatorname{má} x\{|A|,|B|\}}
$$

Ahora bien, la distancia del punto $P$ al foco $F$ de coordenadas $(a, b)$ por la métrica del taxista está dada de la siguiente forma:

$$
d_{t}(P, F)=|x-a|+|y-b|
$$

$Y$ por la Definición 2 se iguala $d_{t}(P, F)$ y $d_{t}(P, l)$ quedando la expresión de la parábola:

$$
\begin{aligned}
d_{t}(P, F) & =d_{t}(P, l) \\
|x-a|+|y-b| & =\operatorname{mín}_{M \in l} d_{t}(P, M) \\
|x-a|+|y-b| & =\frac{|A x+B y+C|}{\operatorname{máx}\{|A|,|B|\}}
\end{aligned}
$$

Algunas representaciones graficas de la parábola en la métrica del taxista son:

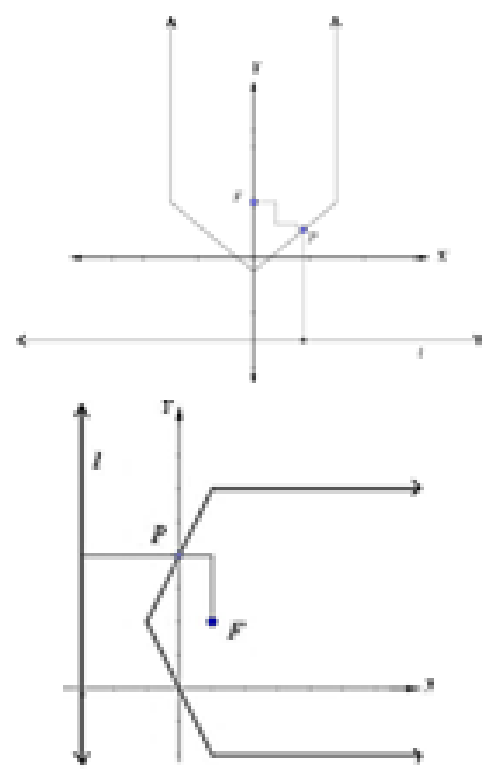

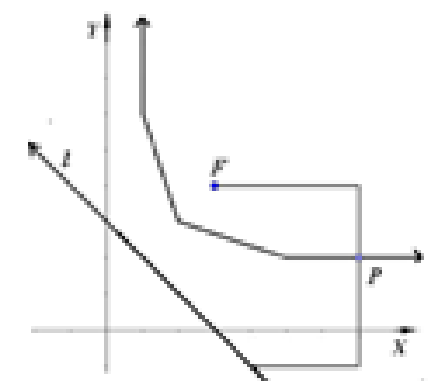

Figura 14. Parábolas en la métrica del taxista.

Fuente: Los autores

Elipse. En el caso de esta cónica, el proceso analítico es más corto y se parte de la Definición 3 , tomándose como focos $F_{1}=(a, b)$ y $F_{2}=(c, d)$. Para el caso de la distancia entre el punto $P$ y el foco $F_{1}$ aplicándole la métrica del taxista quedaría de la siguiente forma:

$d_{t}\left(P, F_{l}\right)=|x-a|+|y-b|$ de forma análoga, la distancia entre $P$ y el foco $F_{2}$ queda:

$$
d_{t}\left(P, F_{2}\right)=|x-c|+|y-d|
$$

la elipse en la métrica del taxista queda definida de forma general como:

$$
\begin{gathered}
d_{t}\left(P, F_{1}\right)+d_{t}\left(P, F_{2}\right)=k \\
|x-a|+|y-b|+|x-c|+|y-d|=k .
\end{gathered}
$$

Algunos ejemplos de representaciones graficas de la elipse son:

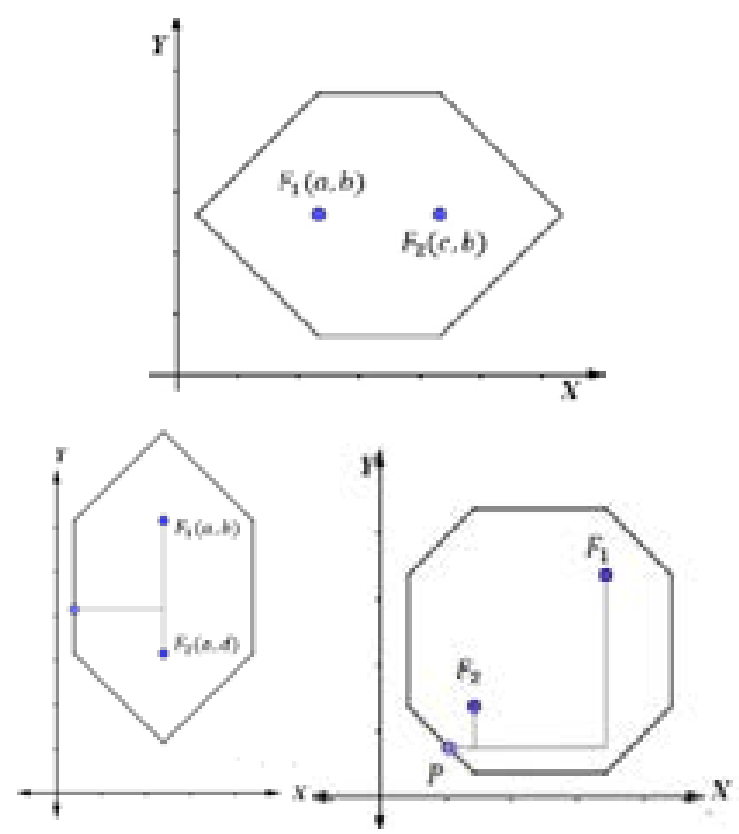

Figura 15. Elipse en la métrica del taxista. Fuente: Los autores 
Hipérbola. De acuerdo a la Definición 4, se nombra $F 1=(a, b)$ y $F 2=(c, d)$ los focos de la hipérbola y $P=(x, y)$ un punto cualquiera que pertenece a ella, por la métrica del taxista, la distancia $d_{t}\left(F_{l}, P\right)$ y $d t\left(F_{2}, P\right)$ están dadas por las siguientes expresiones:

$$
\begin{gathered}
d_{t}\left(F_{1}, P\right)=|x-a|+|y-b|, \\
d_{t}\left(F_{2}, P\right)=|x-c|+|y-d| .
\end{gathered}
$$

Reemplazando las expresiones anteriores por la expresión (4) de la Definición 4, obtenemos la siguiente ecuación:

$$
|| x-a|+| y-b|-(|x-c|+|y-b|)|=k,
$$

es similar a tener:

$$
|| x-a|+| y-b|-| x-c|-| y-b||=k
$$

Una representación en el lenguaje grafico de la hipérbola cuando los focos comparten la misma ordenada es la siguiente.
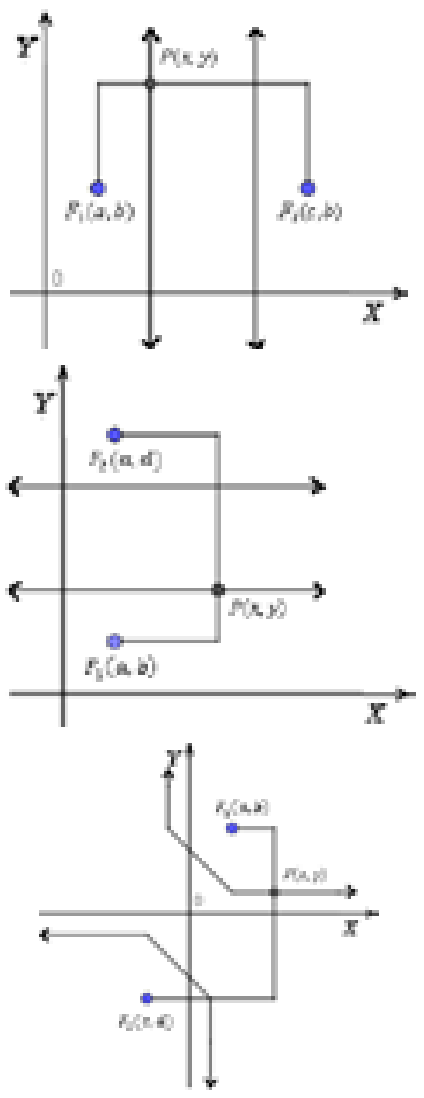

Figura 16. Hipérbola en la métrica del taxista.

Fuente: Los autores

\section{CÓNICAS EN LA MÉTRICA DEL MÁXIMO.}

Circunferencia. El comportamiento geométrico de la circunferencia en la métrica del máximo se ve determinado por la Definición 1, llamaremos $C=(h, k)$ el centro de la circunferencia con radio y $P=(x, y)$ un punto cualquiera que pertenece a la circunferencia, por la métrica del máximo se tiene que la distancia del punto $P$ al centro está dado por:

$$
d_{M}(C, P)=\operatorname{máx}\{|x-h|,|y-k|\},
$$

y por la expresión (1) de la Definición 1, se tiene:

$$
\text { máx }\{|x-h|,|y-k|\}=r,
$$

la gráfica que representa la ecuación de una circunferencia con centro en el punto $C=(h, k)$ es de la siguiente forma:

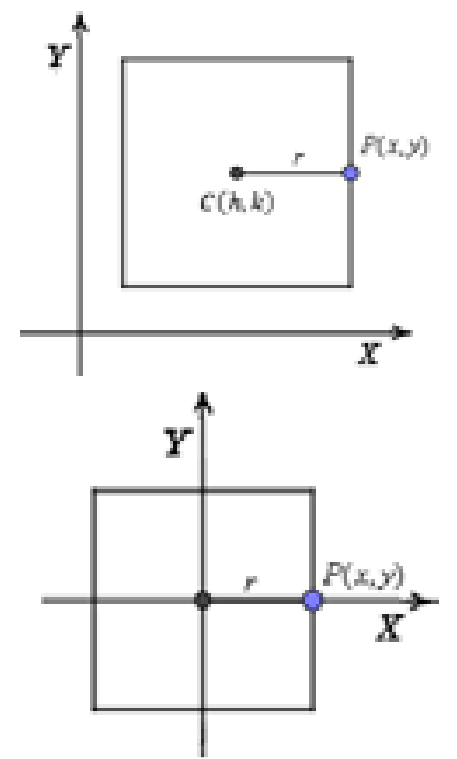

Figura 17. Representación de circunferencias en la métrica del máximo.

Fuente: Los autores.

Parábola. En esta cónica, se realizará un razonamiento muy similar al realizado con la parábola en la métrica del taxista, debido a la gran similitud entre ambas métricas, por tanto, teniendo en cuenta la Definición 2, se debe encontrar una expresión para la distancia de un punto $P$ a una recta $l$ de la forma $A x+B y+C$ en la métrica del máximo. 
Para esto se recurre nuevamente al método de las circunferencias (ver Definición 5), teniendo en cuenta lo anterior se podrían presentar dos casos, el primero (CASO I), cuando la circunferencia con centro en $P$, es tangencial a la recta $l$, por tal razón este radio vendría siendo la distancia mínima entre el punto $A$ y la recta $l$.

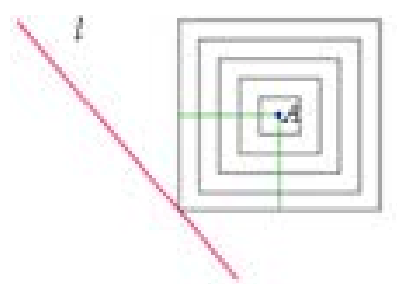

Figura 18. Caso I. Distancia mínima de un punto a una recta en la métrica del máximo.

Fuente: Los autores

El otro caso (CASO II), es más común que el Caso I, y resulta cuando la recta es de sentido horizontal o vertical, en este caso la circunferencia con centro en $A$, intercepta en más de un punto a la recta, pero la distancia mínima, será el radio de dicha circunferencia, como se muestra a continuación:
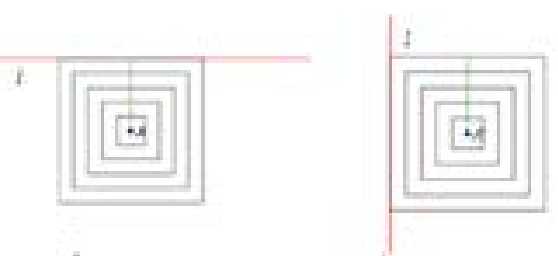

Figura 19. Caso II. Distancia mínima de un punto a una recta, cuando esta tiene rectas horizontales o verticales.

Fuente: Los autores.

Para encontrar una expresión analítica a la distancia mínima que hay entre un punto y una recta en esta métrica, se tomaran los resultados encontrados en la parábola con la métrica del taxista, obteniéndose para el Caso II las siguientes expresiones:

$$
\begin{gathered}
d_{M}(P, l)=\frac{|A x+C|}{|A|},(\text { para una recta vertical }) . \\
d_{M}(P, l)=\frac{|B y+C|}{|B|},(\text { para una recta horizontal }) .
\end{gathered}
$$

En el Caso I, la distancia mínima del punto $P$ de coordenadas $(x, y)$ a la recta estará dado por:

$$
d_{M}\left(P, A_{A \in l}\right)=r \text {, donde } r \text { es el radio de la }
$$

circunferencia en la métrica del máximo con centro en $P(x, y)$ que es tangencial a la recta $l$ en el punto $A$. Teniendo en cuenta la Definición 2 y los casos anteriores, se define $F=(a, b)$ y la directriz de la forma $A x+B y+C=0$, por tanto, los puntos $P$ de coordenadas $(x, y)$ que pertenecen a la parábola satisfacen la siguiente expresión:

$$
\operatorname{má} x\{|x-a|,|y-b|\}=\operatorname{mín}_{A \in l} d_{M}(P, A)
$$

Obteniéndose tres ejemplos de parábola en la métrica del máximo:

\section{*Parábola con directriz oblicua:}

máx $\{|x-a|,|y-b|\}=r$, donde $r$ es el radio de una circunferencia con centro en $P(x, y)$ que es tangencial a la recta $l$ y que pasa por el foco $F(a, b)$

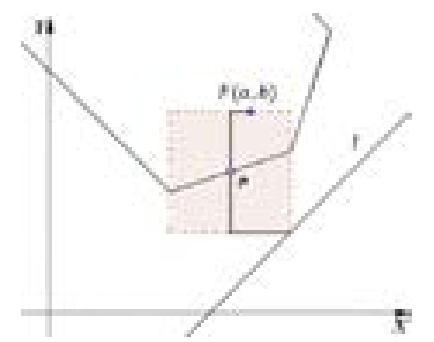

Figura 20. Parábola en la métrica del máximo con directriz oblicua. Fuente: Los autores.

\section{*Parábola con directriz vertical:}

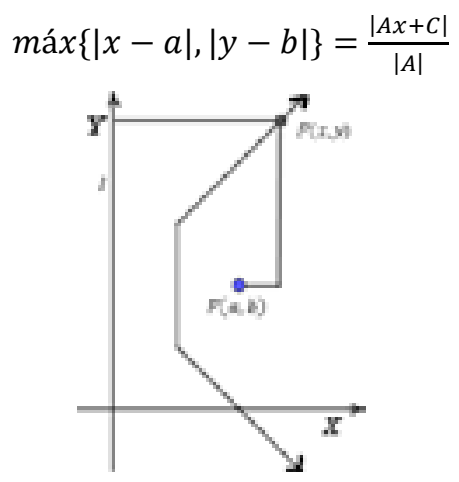

Figura 21. Parábola en la métrica del máximo con directriz vertical.

Fuente: Los autores. 
*Parábola con directriz horizontal:

.máx $\{|x-a|,|y-b|\}=\frac{|B y+C|}{|B|}$

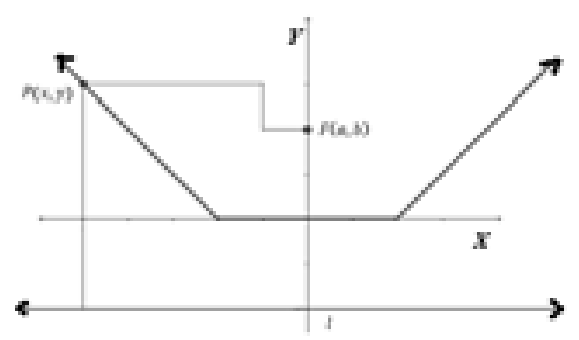

Figura 22. Parábola en la métrica del máximo con directriz horizontal.

Fuente: Los autores.

Elipse. Sean $F_{1}$ y $F_{2}$ de coordenadas $(a, b)$ y $(c, d)$ respectivamente, focos de la elipse y sea $k$ una constante que satisface la expresión $k>d_{M}\left(F_{1}, F_{2}\right)$, por la Definición 3, los puntos $P$ de la forma de $(x, y)$ un plano pertenecerán a la elipse en la métrica del máximo si satisface:

$$
d_{M}\left(P, F_{1}\right)+d_{M}\left(P, F_{2}\right)=k
$$

Y por la métrica del máximo se tiene:

$$
\begin{aligned}
& d_{M}\left(P, F_{1}\right)=\operatorname{máx}\{|x-a|,|y-b|\} \\
& d_{M}\left(P, F_{2}\right)=\operatorname{má} x\{|x-c|,|y-d|\}
\end{aligned}
$$

Quedando determinada la ecuación de la elipse de la siguiente forma:

máx $\{|x-a|,|y-b|\}+\operatorname{má} x\{|x-c|,|y-d|\}=k$

A continuación, se muestra algunos ejemplos de elipse en la métrica del máximo.
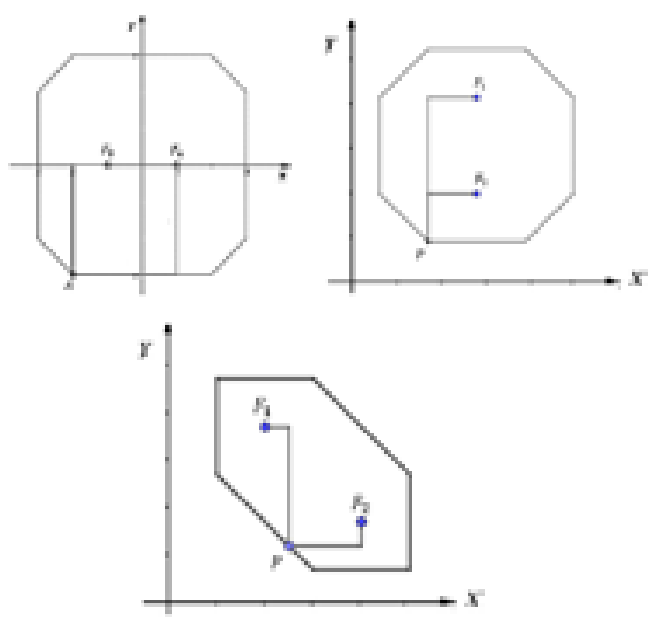

Figura 23. Elipse en la métrica del máximo. Fuente: Los autores.
Hipérbola. De acuerdo a la Definición 4 se estudia la hipérbola asumiendo $F_{1}$ y $F_{2}$ de coordenadas $(a, b)$ y $(c, d)$ respectivamente, los focos de la hipérbola y $P$ de coordenadas $(x, y)$ un punto cualquiera que pertenece a ella, el punto $P$ debe satisfacer la expresión (4) y teniendo en cuenta la métrica del máximo, las distancias y $d_{M}\left(F_{1}, P\right) Y d_{M}\left(F_{2}, P\right)$ están dadas por las siguientes expresiones:

$$
\begin{aligned}
& d_{M}\left(F_{1}, P\right)=\operatorname{máx}\{|x-a|,|y-b|\} \\
& d_{M}\left(F_{2}, P\right)=\operatorname{máx}\{|x-c|,|y-d|\} .
\end{aligned}
$$

Obteniendo la siguiente ecuación de hipérbola en la métrica del máximo:

$|\operatorname{má} x\{|x-a|,|y-b|\}-\operatorname{máx}\{|x-c|,|y-b|\}|=k$,

A continuación, se muestran algunas representaciones graficas de la hipérbola en la métrica del máximo.

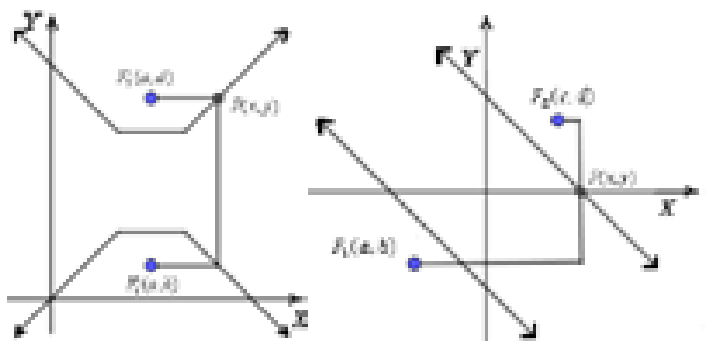

Figura 24. Hipérbolas en la métrica del máximo. Fuente: Los autores.

\section{CÓNICAS EN LA MÉTRICA DISCRETA}

Circunferencia. De acuerdo a la métrica discreta, y la expresión (1) de la Definición 1, los únicos valores de interés para $r$ son $r=0$ y $r=1$, por esta razón se evalúa cada uno de los casos de manera separada.

Caso I: $r=0$

Para que la distancia entre dos puntos en la métrica discreta sea igual a 0 , es necesario que los puntos de los cuales vamos a medir las distancias sean iguales: $d_{d}(C, P)=0$, lo que quiere decir que:

$$
C(h, k)=P(x, y) \rightarrow h=x \wedge k=y .
$$


La única forma para que se cumpla este caso es que el centro de la circunferencia sea igual a los puntos que la conforman, por lo cual la gráfica tendrá la siguiente estructura:

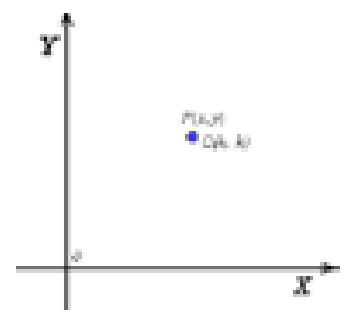

Figura 25. Representación de la circunferencia con centro en $(h, k)$ y $(x, y)$ con $(h, k)=.(x, y)$

Fuente: Los autores.

Caso II: $r=1$

Para que la distancia entre dos puntos en la métrica discreta sea igual a 1 , es necesario que los puntos de los cuales vamos a medir las distancias sean diferentes $d_{d}(C, P)=1$, por lo cual: $C(h, k) \neq P(x, y) \rightarrow h \neq x \vee k \neq y$, en este caso la gráfica de la circunferencia es completamente opuesta al caso anterior, por lo cual la gráfica de la circunferencia en este caso tendrá la siguiente estructura:

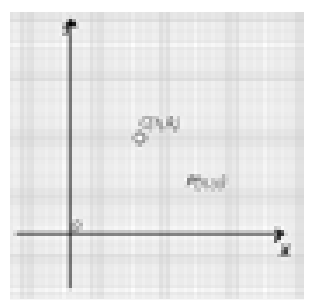

Figura 26. Representación de la circunferencia en la métrica discreta cuando $C(h, k)$ es diferente de $P(x, y)$.

Fuente: Los autores.

Parábola. Para obtener una aproximación a la expresión analítica y gráfica de esta cónica en la métrica discreta, se debe definir una la recta $l$ y un punto $F$ de coordenadas $(a, b)$ que como anteriormente se ha mencionado, es el foco de la parábola, y no pertenece a la recta $l$, ahora recurriendo a la Definición 2 y adaptada a esta métrica se tiene: $d_{d}(P, F)=d_{d}(P, l)$ pero recurriendo a la métrica discreta las únicas longitudes de distancia que son posibles en esta métrica son cero y uno, por lo que se tienen dos casos:

$$
\begin{aligned}
& \text { Caso I: } d_{d}(P, F)=d_{d}(P, l)=1 . \\
& \text { Caso II: } d_{d}(P, F)=d_{d}(P, l)=0 .
\end{aligned}
$$

Para el caso I, la única forma de que $d_{d}(P, F)=1$ es que el punto $P$ sea distinto al punto $F$

$(P \neq F)$, en este orden de ideas, todos los puntos del plano a excepción del foco $F$ cumplen que la distancia sea igual a uno, ya que si $P=F$ entonces $d_{d}(F, F)=0$, este resultado se ilustra a continuación:

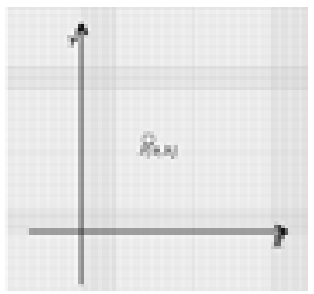

Figura 27. Puntos cuya distancia $F$ a es uno en la métrica discreta.

Fuente: Los autores.

El fondo subrayado del anterior grafico hace referencia a todos los puntos del plano, y la representación del punto $F$ en blanco hace referencia a que ese espacio es vacío. De forma similar, todos los puntos $P$ a excepción de los que pertenecen a la recta $l$, satisfacen que $d_{d}$ $(P, l)=1$, ya que si el punto $P$ está sobre la recta $l$ entonces $P \in l$ ! y la distancia del punto $P$ a la recta $l$ es $d_{d}(P l)=0$, por tal razón la gráfica queda de la siguiente forma: 


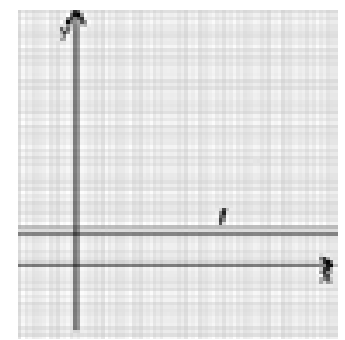

Figura 28. Puntos cuya distancia a la recta $l$ es igual a uno en la métrica discreta.

Fuente: Los autores.

Juntando los dos resultados anteriores:

$d_{d}(P, F)=1 y d_{d}(P, l)=1, \mathrm{y}$, nos queda que todos los puntos del plano excepto el foco y los que pertenecen a la recta satisfacen que en ambos casos (distancia al foco y a la recta) sea igual a uno, dicho de otra forma:

$d_{d}(A, F)=d_{d}(A, l)=1$ para $\forall A \in \mathbb{R}^{2}-\{F \wedge l\}$.

Lo anterior quedaría ilustrado de la siguiente forma:

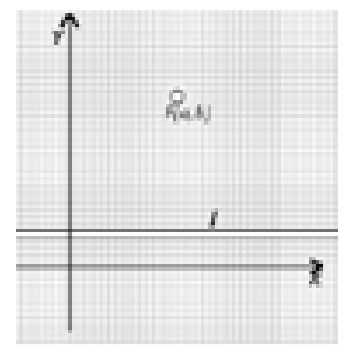

Figura 29. Parábola en la métrica discreta cuando todas las distancias es igual a uno.

Fuente: Los autores.

Ahora para el caso II se tiene que

$d_{d}(P, F)=d_{d}(P, l)=0$, esta expresión se dará si $P=F$ y aparte de esto pertenecer a la recta $l$, lo que implicaría que el foco pertenece a la recta directriz, incumpliéndose la condición de parábola, por tal razón solo el caso I es posible.

Elipse. Teniendo en cuenta la expresión propuesta por la Definición 3, sean $P=(x, y)$, puntos que pertenecen a la elipse, $F_{1}, F_{2}$, de coordenadas $(a, b)$ y $(c, d)$ sus focos, y $k$ una constante, aplicando la notación de distancia discreta se tiene:

$$
d_{d}\left(P, F_{1}\right)+d_{d}\left(P, F_{2}\right)=k,
$$

pero por la métrica discreta las únicas distancias posibles son uno y cero, es decir:

$d_{d}\left(P, F_{1}\right)=0$ si $P=F_{1}$ ó $d_{d}\left(P, F_{1}\right)=1$ si $P \neq F_{1}$, de igual forma:

$d_{d}\left(P, F_{2}\right)=0$ si $P=F_{2}$ ó $d_{d}\left(P, F_{2}\right)=1$ si $P \neq F_{2}$, ahora reemplacemos las expresiones anteriores en la expresión algebraica de la Definición 3 y obtenemos cuatro resultados:

$$
\begin{aligned}
& 1+1=2 \\
& 1+0=1 \\
& 0+1=1 \\
& 0+0=0
\end{aligned}
$$

Teniendo en cuenta la expresión $k>d_{d}\left(F_{1}, F_{2}\right)$, pero como $d_{d}\left(F_{1}, F_{2}\right)=1, k>1$, por tanto, de los cuatro posibles casos, solo servirá el primer caso: $1+1=2$

De lo anterior, los puntos $P$ que satisfacen $d_{d}\left(P, F_{1}\right)=1$, es todo el plano sin el foco $F$, igualmente, para que $d_{d}\left(P, F_{2}\right)$ sea igual a 1 , necesariamente el punto $P$ debe ser distinto al foco $F_{2}$, es decir que todo el plano sin el foco $F_{2}$ cumplen esta expresión, uniendo los dos anteriores resultados, se tiene que la elipse en la métrica discreta es el conjunto de los puntos $P$ tales que: $P \in \mathbb{R}^{2}-\left\{F_{1}, F_{2}\right\}$.

Esto gráficamente representado es:

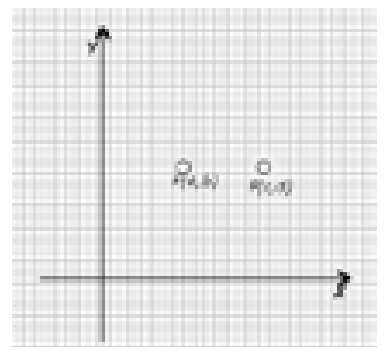

Figura 30. Elipse en la métrica discreta

Fuente: Los autores.

De esta gráfica se puede detallar que esta rellenado todo el plano a excepción de la 
representación en blanco de los dos puntos fijos, llamados focos de la elipse.

Hipérbola. Nos remitiremos a la Definición 4 y sean $F_{1}, F_{2}$, los focos de la hipérbola de la forma $(a, b),(c, d)$ respectivamente, y sea $(x, y)$ un punto $P$ que pertenezca a la hipérbola, de acuerdo a la expresión (4) y la métrica discreta la distancia entre dos puntos, solo puede ser igual a cero o a uno, por lo cual evaluaremos los siguientes casos:

$$
\begin{aligned}
& \left|d_{d}\left(F_{1}, P\right)\right|=0, \text { si } F_{1}=P . \\
& \left|d_{d}\left(F_{1}, P\right)\right|=1, \text { si } F_{1} \neq P . \\
& \left|d_{d}\left(F_{2}, P\right)\right|=0, \text { si } F_{2}=P . \\
& \left|d_{d}\left(F_{2}, P\right)\right|=1, \text { si } F_{2} \neq P .
\end{aligned}
$$

Reemplazando los ítems anteriores en la expresión (4) de la Definición 4, tenemos cuatro casos posibles para evaluar:

$$
\begin{aligned}
& \text { Caso I, cuando } F_{1}=P \text { y } F_{2}=P, \\
& \text { Caso II, cuando } F_{1} \neq P \text { y } F_{2}=P, \\
& \text { Caso III, cuando } F_{1}=P \text { y } F_{2} \neq P, \\
& \text { Caso IV, cuando } F_{1} \neq P_{\text {y }} F_{2} \neq P,|0|-|1| \mid=k \text {, entonces } k=0 \\
& \qquad\|1|-| 1\|=k \text {, entonces } k=1
\end{aligned}
$$

Hemos evaluado cuatro casos de acuerdo a las posibilidades de distancia en esta métrica, y se ha encontrado que no hay ninguna forma valida, ya que por un lado, se descartan los casos cuando $k=0$, debido a que $k$ es positivo, y cuando $k=1$, porque se debe cumplir que la constante sea menor a la distancia de los dos focos $\left(k<d_{d}\left(F_{1}, F_{2}\right)\right.$, y en este caso resulta ser igual. Por tal razón se concluye que la hipérbola no está definida en la métrica discreta.

\section{DISCUSIÓN}

Las Cónicas en otras métricas, una actividad matemática analizada desde la teoría de los registros de representación semiótica

La teoría de los registros de representación semiótica propuesto por Duval manifiesta que “lo que primero importa para la enseñanza de las matemáticas no es la elección del mejor sistema de representación sino lograr que los estudiantes sean capaces de relacionar muchas maneras de representar los contenidos matemáticos" (Duval, 2006, p. 158), en el desarrollo de la investigación de las cónicas, se pudo evidenciar que al variar la forma de medir hay un cambio en las condiciones analíticas y geométricas de las nuevas figuras, por lo tanto, es importante el papel de las métricas en cuanto a deducciones de lugares geométricos se refiere.

Si estudiamos los textos de matemáticas en donde se expone la temática de las cónicas, se evidencia un enfoque desde la geometría analítica, es decir que se quiere estudiar estas figuras geométricas desde técnicas de análisis matemático y algebra, no significa que este mal enfocado, pero es la forma tradicional en la que siempre se enseña esta temática, es decir todo se resume a identificar los elementos de cada figura (vértice, focos, excentricidad etc.) y luego 
a realizar tratamientos con diferentes tipos de ecuaciones (ecuación canónica), convirtiéndose en una actividad mecánica de manipular una ecuación, realizar una tabla de valores y graficar dejando de lado el concepto de lugar geométrico implícito en estas figuras y con ello el análisis de tipo intuitivo y práctico que puede trabajarse al momento de abordar estos objetos matemáticos.

Un gran interrogante de los profesores de matemáticas y que es motivo de investigaciones en el campo de la educación matemática es ¿qué tipos de problemas seleccionar para desarrollar el interés de los alumnos y favorecer la adquisición de conocimientos matemáticos?" (Duval, 2006, p. 143), particularizando este cuestionamiento al aprendizaje de las cónicas, en la presente investigación se mostró una forma de abordar estas figuras con el tratamiento de diferentes métricas distintas a la usual, esta actividad dejo ver como a través de cambios de registros de representación verbales lenunciado de lugares geométricos), analíticos (expresiones algebraicas de las cónicas en diferentes métricas) y geométricos (Construcción de figuras) se puede priorizar el concepto de lugar geométrico así como dar cabida a razonamientos de tipo practico e intuitivo para el estudio de las figuras, porque basto con entender como era el proceso de medida en cada métrica así como las definiciones de lugar geométrico para poder encontrar distintas representaciones de las cónicas y detallar las relaciones y diferencias en la construcción de esta actividad matemática.
Para Duval (2006), la actividad matemática necesariamente se remite a contextos de representación y una actividad matemática correctamente desarrollada debería llevar al estudiante a ser capaz de reconocer el mismo objeto matemático en diferentes contextos, lo que provocaría procesos cognitivos como la transformación y la coordinación interna. En distintas investigaciones se ha manifestado que el aprendizaje de un concepto matemático es más eficaz cuando se trabaja desde distintas representaciones, pues la matemática al movilizarse en lo abstracto necesita del apoyo de diferentes medios para poder ser comprendida.

En cuanto a la transformación y la coordinación interna, procesos cognitivos que se mencionaron anteriormente, el primero hace referencia a dos momentos fundamentales al realizar una actividad matemática: tratamiento y conversión; mientras que el segundo (coordinación interna) hace referencia a la actividad cognitiva donde el individuo selecciona el registro de representación adecuado para dar solución a un problema.

A continuación, se expone un ejemplo de cómo el primer proceso cognitivo (transformación) se manifestó en la investigación, para ello se traerá el caso la parábola (el diagrama es similar para las otras tres cónicas) 


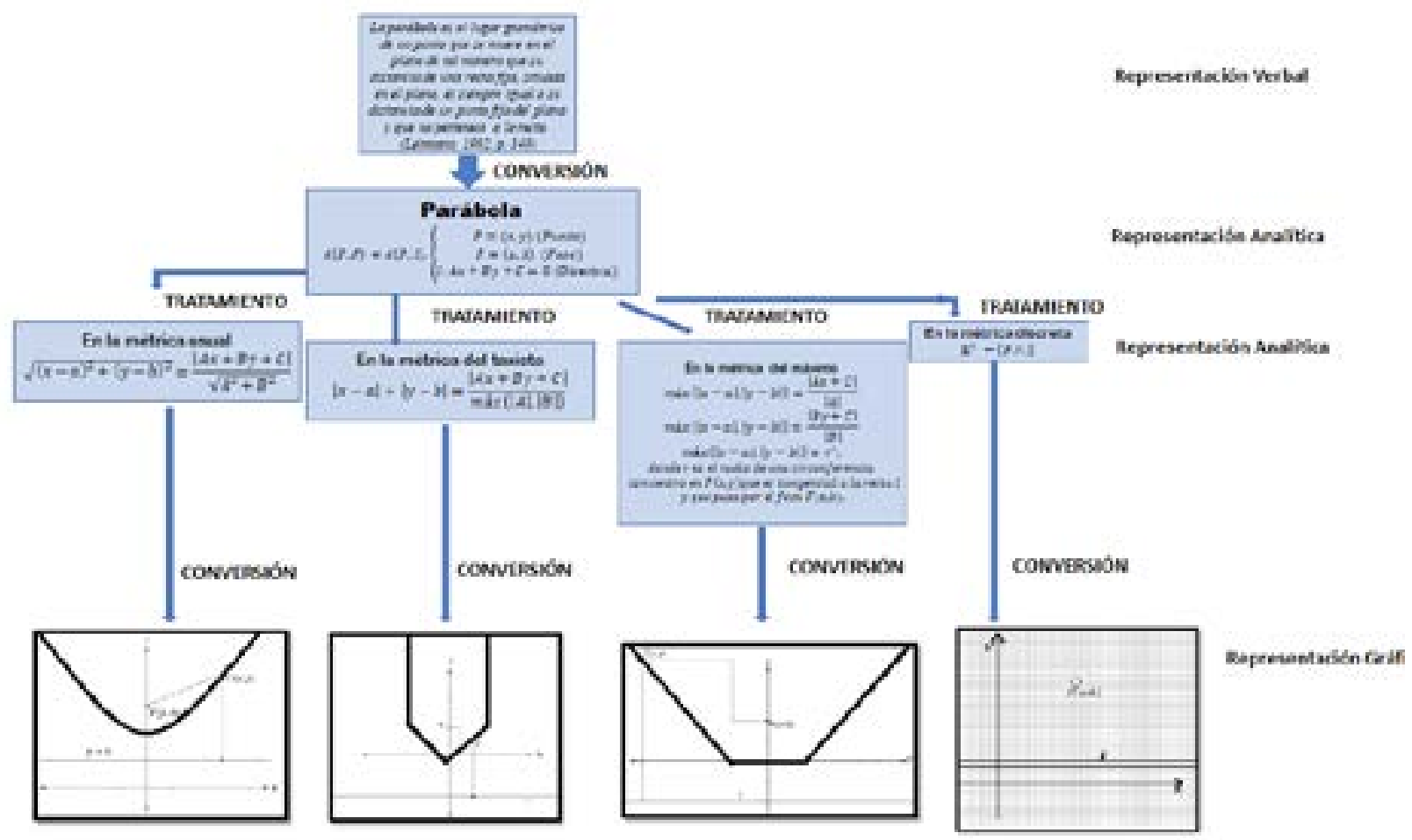

Figura 31. Alternancia de la conversión y el tratamiento en la resolución del lugar geométrico de la Cónica desde diferentes métricas.

\section{Fuente: Elaboración propia}

En el diagrama se puede detallar en un primer momento un registro de representación verbal que es la definición como lugar geométrico de la parábola, después de leer el enunciado se deduce una expresión algebraica que generaliza los principales aspectos del mismo, en este punto ocurre una conversión de registro: de verbal a analítico. Luego de tener una expresión simbólica general y teniendo en cuenta las definiciones de métrica usual, del taxi, del máximo y discreta se realiza un tratamiento de la expresión simbólica a otra expresión algebraica que reúne tanto las características de lugar geométrico de la parábola como la definición de la métrica correspondiente, finalmente con una expresión algebraica establecida (representación verbal) se procede a realizar una conversión de registro: del analítico a gráfico. Ahora bien, este análisis no solo puede ser en sentido vertical, también analizándolo de manera horizontal podemos detallar los diferentes tratamientos de la parábola tanto desde un registro analítico como de un registro gráfico.

Lo anterior tan solo es un ejemplo del tipo de análisis que se realizó para lograr las cónicas en las diferentes métricas y se puede observar los diferentes tratamientos y conversiones para obtener las representaciones algebraicas y geométricas, esto muestra algunas de las bondades que ofrece el analizar la geometría desde diferentes métricas cuando el objetivo del aprendizaje es rescatar la matemática intuitiva como es el caso de los lugares geométricos en las cónicas.

Esta investigación sirve de ejemplo de cómo abordar la enseñanza y el aprendizaje de las cónicas dando prioridad al lugar geométrico, pues "la comprensión no significa dar un salto desde el contenido de una representación hasta el concepto puramente matemático representado sino en relacionar diversos contenidos de representación del mismo concepto" (Duval, 
2006, p. 158), pues se aborda cada cónica con diferentes representaciones (verbal, analítica y gráfical y se muestra de forma detallada como lograr los diferentes tratamientos y conversiones para llegar al conocimiento esperado.

\section{CONCLUSIONES}

En muchas ocasiones se asocia a una cónica con una representación gráfica determinada, en esta investigación se lograron nuevas representaciones tanto algebraicas como geométricas de las cónicas cuando se cambia la forma de medir, con esto entendemos mejor la definición de cada cónica como lugar geométrico, dejando claro que hay varios caminos para abordar las cónicas en un proceso de enseñanza escolar no solo desde la geometría analítica.

El trabajo investigativo se centró especialmente en el tratamiento y la conversión de las representaciones provocando coordinaciones en los diferentes registros semióticos, según la teoría de los registros de representación, este tipo de actividad provoca una mejor compresión del conocimiento, aparte de esto integra diferentes tareas como la comprensión lectora lenunciados de los lugares geométricos de las cónicas), interpretación simbólica y ejercitación del pensamiento espacial

Posiblemente la situación más importante en el tratamiento de las cónicas lo constituyen las diferentes representaciones obtenidas, pues se encontró nueva información (como las ecuaciones o las gráficas), lográndose visualizar diferentes propiedades que generalmente no se contemplan al momento de aprender este conocimiento y obteniendo un constructo del concepto de cónica más amplio.

Las cuádricas vendrían siendo la representación tridimensional de las cónicas, partiendo del trabajo realizado con las cónicas en otras métricas, se podría estudiar la relevancia que tienen estas métricas en las cuádricas debido a su estrecha relación con las cónicas, y partiendo de la teoría de Registros de representación se podría desarrollar una actividad matemática similar donde se analicen las cuadricas como lugares geométricos desde diferentes métricas.

\section{REFERENCIAS BIBLIOGRÁFICAS}

Antonio, J. \& Garzón, C. (2017). Estudio geométrico y analítico de las cónicas en algunas métricas. (Tesis de Pregrado). Universidad Pedagógica y Tecnológica de Colombia. Tunja, Colombia.

Bonilla, B., González, P., \& Chavarro, S. (2014). Las cónicas en la geometría del taxista: una propuesta didáctica desde la teoría de los modos de pensamiento. Acta Latinoamericana de Matemática Educativa, Vol. 27, 666-673

Bonilla, D (2012). La Elipse desde la perspectiva de la Teoría de los Modos de Pensamiento. (Tesis de maestría). Pontificia Universidad católica de Valparaíso, Valparaíso, Chile.

Duval, R. (2006). Un tema crucial en la educación matemática: La habilidad para cambiar el registro de representación. La Gaceta de la Real Sociedad Matemática Española, 9 (1), 143-168.

Izquierdo, C. \& Ardila, P. (2013). Estudio de la métrica de manhattan. Segmentos, rectas, rayos, circunferencias y algunos lugares geométricos en la geometría del taxista. (Tesis de Pregrado) Universidad Pedagógica Nacional, Bogotá, Colombia.

Lehmann, C. (1992). Geometría Analítica. México: LIMUSA. Lima: UNMSM.

Loiola, G., \& Costa, S. (2015). As Cônicas na Geometria do Taxi. Ciência e Natura. Revista do Centro de Ciências Naturais e Exatas - UFSM, p.179-191. 
Ministerio de Educación Nacional, (1998). Matemáticas: Lineamientos curriculares (Publicación ISBN/ISSN/DL N 958-691067-9). Colombia: El ministerio.

Ministerio de Educación Nacional, (2016). Derechos básicos de aprendizaje. Matemáticas. V2: El ministerio.

Munkres, J. (2002). Topología. Madrid, España: Prentice Hall.

Valdivia, C., \& Parraguez, M. (2012). Evolución cognitiva del concepto parábola como lugar geométrico: una mirada desde la teoría APOE. Acta Latinoamericana de Matemática Educativa 25. p. 593-601.

Vasco, C. E. (2006). Didáctica de las matemáticas: artículos selectos. U. Pedagógica Nacional. 